REPORTS ON MATHEMATICAL LOGIC

53 (2018), 97-109

doi: $10.4467 / 20842589 R M .18 .006 .8839$

Yoshihito TANAKA

\title{
A CUT-FREE PROOF SYSTEM FOR A PREDICATE EXTENSION OF THE LOGIC OF PROVABILITY
}

\begin{abstract}
A b s t $\mathrm{r}$ a c t. In this paper, we introduce a proof system NQGL for a Kripke complete predicate extension of the logic GL of provability. While $\mathbf{G L}$ is defined by $\mathbf{K}$ and the Löb formula $\square(\square p \supset p) \supset \square p$, NQGL does not have the Löb formula as its axiom, but has a non-compact rule, that is, a derivation rule with countably many premises, instead. We show that NQGL enjoys cut admissibility and is complete with respect to the class of Kripke frames such that for each world, the supremum of the length of the paths from the world is finite.
\end{abstract}

Received 21 February 2018

Keywords and phrases: Provability logic, predicate logic, cut-free system.

AMS subject classification: 03F05, 03F 45. 


\section{Introduction}

In this paper, we introduce a cut-free proof system for a Kripke complete predicate extension of $\mathbf{G L}$, where $\mathbf{G L}$ is a propositional normal modal logic defined by $\mathbf{K}$ and the Löb formula

$$
\square(\square p \supset p) \supset \square p .
$$

GL is well-known as the logic of provability, in the sense that a propositional modal formula $\phi$ is in GL if and only if $f(\phi)$ is provable in the Peano arithmetic PA for every arithmetical interpretation $f$ (e.g. [3]).

A Kripke frame $(W, R)$ is said to be conversely well-founded, if there exists no countably infinite list $\left(w_{i}\right)_{i \in \mathbb{N}}$ of elements of $W$ which satisfies $\left(w_{i}, w_{i+1}\right) \in R$ for any $i \in \mathbb{N}$, and is said to be of bounded length, if for any $w \in W$ the supremum of the length of the lists $w_{0}, w_{1}, \ldots, w_{n}$ which satisfy $\left(w_{i}, w_{i+1}\right) \in R$ and $w_{0}=w$ is finite. We write $\mathfrak{F} \mathfrak{I}, \mathfrak{B} \mathfrak{L}$, and $\mathfrak{C} \mathfrak{W}$ for the classes of transitive Kripke frames which are finite and irreflexive, of bounded length, and conversely well-founded, respectively. For any class $C$ of Kripke frames, we write $\mathbf{M P}(C)$ and $\mathbf{M Q}(C)$ for the sets of propositional modal formulas and predicate modal formulas which are valid in $C$, respectively. It is known (e.g. [3]) that

$$
\mathbf{G L}=\operatorname{MP}(\mathfrak{F} \mathfrak{I})=\operatorname{MP}(\mathfrak{C W}) .
$$

Therefore,

$$
\mathbf{G L}=\operatorname{MP}(\mathfrak{B} \mathfrak{L}) .
$$

However, the situation in predicate extensions of GL is not so clear. Let QGL be the smallest predicate normal modal logic which includes GL as its propositional fragment. Let $\mathrm{QPL}(\mathrm{PA})$ be the set of predicate modal formulas defined by

$$
\mathbf{Q P L}(\mathrm{PA})=\{\phi \mid \mathrm{PA} \vdash f(\phi) \text { for every interpretation } f\} .
$$

It is shown in [8] that QGL $\varsubsetneqq \mathbf{M Q}(\mathfrak{C W})$ and QGL is incomplete with respect to any classes of Kripke frames. It is also proved in [8] that QGL $\varsubsetneqq$ QPL(PA), that is, QGL is arithmetically sound but incomplete, and $\mathbf{Q P L}(\mathrm{PA}) \nsubseteq \mathbf{M Q}(\mathfrak{F} \mathfrak{I})$. Subsequently, [1] shows that if a closed predicate modal formula $\phi$ is not valid in a finite irreflexive Kripke model with finite domains then there exists an interpretation $f$ such that PA $\forall f(\phi)$, 
and [6] shows that MQ(CW) $\nsubseteq \mathbf{Q P L}(\mathrm{PA})$. To summarize these results, we have the following:

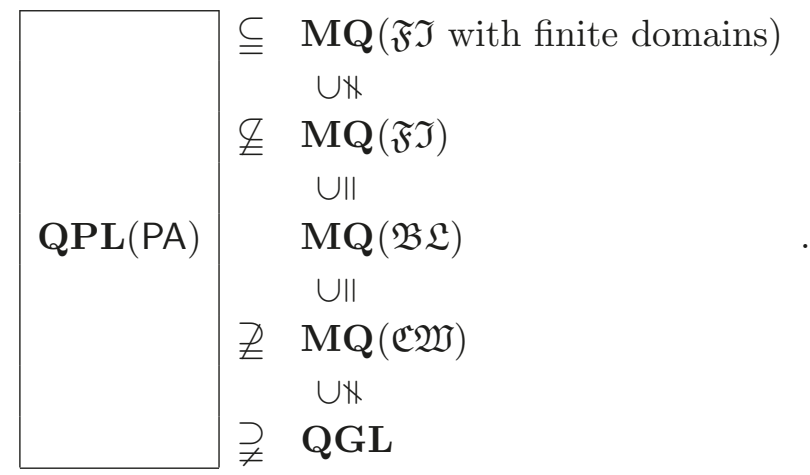

On the other hand, [15] introduces a logic $\mathbf{Q} \mathbf{G L}^{b}$, a predicate extension of $\mathbf{G L}$, in which all occurrences of individual variables in a scope of a modal operator are considered to be bound, and

$$
\square \phi \rightarrow \square \forall x \phi
$$

is an axiom schema. It is proved in [15] that $\mathbf{Q} \mathbf{G L}^{b}$ is both arithmetically complete and Kripke complete with respect to $\mathfrak{F} \mathfrak{I}$, under the above restriction in the construction of formulas.

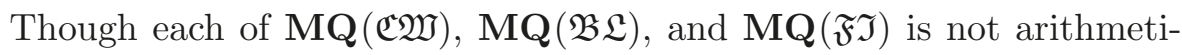
cally sound nor complete as described in (2), it could be of some interest as a problem of pure modal logic to give a cut-free proof system for a Kripke complete predicate extension of $\mathbf{G L}$ without any restriction in the construction of formulas. In this paper, we introduce a proof system NQGL, which is a modal extension of Gentzen's sequent calculus LK for predicate logic, and show the admissibility of the cut-rule and Kripke completeness with respect to $\mathfrak{B L}$. A sequent system for GL is introduced in [7], by means of the following rule for modal operator:

$$
\frac{\square \Gamma, \Gamma, \square \phi \rightarrow \phi}{\square \Gamma \rightarrow \square \phi} .
$$

A proof of the cut-elimination theorem of this system is given in [13] by a syntactic method, and a semantic proof is given in [2]. However, it is proved in [2] that the simple predicate extension of this system does not admit cut-elimination. For a predicate extension with a restriction in the 
construction of the formulas, a cut-free proof system for $\mathbf{Q} \mathbf{G} \mathbf{L}^{b}$ is introduced in [9], by extending the system of [7]. On the other hand, the system NQGL does not include (1) nor (3), although the propositional fragment of NQGL axiomatizes GL. Instead, it has a non-compact rule, that is, a derivation rule with countably many premises. In [4] and [10], a general theory for model existence theorem for propositional modal logic with non-compact rules is given, also, in [11], for their predicate extension with Barcan formula

$$
\mathrm{BF}=\forall x \square \phi \supset \square \forall x \phi .
$$

Kripke completeness of the system defined by NQGL and BF follows from the main theorem of [11].

The outline of the paper is the following: In Section 2, we give basic definitions for syntax and semantics. In Section 3, we introduce the system NQGL. In Section 4, the notions of finitely consistent pairs and saturated pairs are introduced. In Section 5, we show Kripke completeness of NQGL with respect to $\mathfrak{B} \mathfrak{L}$, as well as the admissibility of the cut-rule.

\section{Preliminaries}

The language we consider consists of the following symbols:

1. a countable set $\mathcal{V}$ of variables;

2. $\top$ and $\perp$;

3. logical connectives: $\wedge, \neg, \supset$;

4. quantifier: $\forall$;

5. for each $n \in \mathbb{N}$, countably many predicate symbols $P, Q, R, \cdots$ of arity $n$;

6. modal operator $\square$.

The set $\Phi(\mathcal{V})$ of formulas over $\mathcal{V}$ is the smallest set which satisfies:

1. $\top$ and $\perp$ are in $\Phi(\mathcal{V})$; 
2. if $P$ is a predicate symbol of arity $n$ and $x_{1}, \ldots, x_{n}$ are variables in $\mathcal{V}$ then $P\left(x_{1}, \ldots, x_{n}\right)$ is in $\Phi(\mathcal{V})$;

3. if $\phi$ and $\psi$ are in $\Phi(\mathcal{V})$ then $(\phi \wedge \psi)$ and $(\phi \supset \psi)$ are in $\Phi(\mathcal{V})$;

4. if $\phi \in \Phi(\mathcal{V})$ then $(\neg \phi)$ and $(\square \phi)$ are in $\Phi(\mathcal{V})$;

5. if $\phi \in \Phi(\mathcal{V})$ and $x \in \mathcal{V}$ then $(\forall x \phi) \in \Phi(\mathcal{V})$.

As usual, $\vee$ and $\exists$ are the duals of $\wedge$ and $\forall$, respectively. The symbol $\diamond$ is an abbreviation of $\neg \square \neg$, and for each $n \in \mathbb{N}, \square^{n}$ and $\diamond^{n}$ denote $n$-times applications of $\square$ and $\diamond$, respectively. For each set $S$ of formulas, we write $\square S$ and $\square^{-1} S$ for the sets

$$
\square S=\{\square \phi \mid \phi \in S\}, \quad \square^{-1} S=\{\phi \mid \square \phi \in S\}
$$

of formulas, respectively. For each formula $\phi$, we write $\operatorname{Var}(\phi)$ for the set of variables which have some free or bound occurrences in $\phi$. For each set $S$ of formulas, $\operatorname{Var}(S)$ denotes the set $\bigcup_{\phi \in S} \operatorname{Var}(\phi)$. For each subset $\mathcal{U}$ of $\mathcal{V}$,

$$
\Phi(\mathcal{U})=\{\phi \in \Phi(\mathcal{V}) \mid \operatorname{Var}(\phi) \subseteq \mathcal{U}\} .
$$

A Kripke frame is a pair $(W, R)$, where $W$ is a non-empty set and $R$ is a binary relation on $W$. A system of domains over a frame $F=(W, R)$ is a family $D=\left(D_{w}\right)_{w \in W}$ of non-empty sets such that for all $w_{1}$ and $w_{2}$ in $W$,

$$
\left(w_{1}, w_{2}\right) \in R \Rightarrow D_{w_{1}} \subseteq D_{w_{2}} .
$$

A predicate Kripke frame over $F=(W, R)$ is a triple $(W, R, D)$, where $D$ is a system of domains over $F$. A Kripke model is a four tuple $(W, R, D, I)$, where $(W, R, D)$ is a predicate Kripke frame and $I$ is a mapping called an interpretation which maps each pair $(w, P)$, where $w$ is a member of $W$ and $P$ is an $n$-ary predicate symbol, to an $n$-ary relation $I(w, P) \subseteq\left(D_{w}\right)^{n}$ over $D_{w}$. The relation $\models$ among a Kripke model $M=(W, R, D, I)$, a world $w \in W$, and a closed formula $\phi$ is defined inductively as follows:

1. $M, w \models \top, M, w \not \models \perp$;

2. for any predicate $P$ of arity $n$, $M, w \models P\left(d_{1}, \ldots, d_{n}\right) \Leftrightarrow\left(d_{1}, \ldots, d_{n}\right) \in I(w, P) ;$ 
3. $M, w \models \phi \wedge \psi \Leftrightarrow M, w \models \phi$ and $M, w \models \psi$;

4. $M, w \models \phi \supset \psi \Leftrightarrow M, w \mid \models \phi$ or $M, w \mid=\psi$;

5. $M, w=\neg \phi \Leftrightarrow M, w \not \models \phi$;

6. $M, w \models \forall x \phi \Leftrightarrow M, w \models \phi[d / x]$ for any $d \in D_{w}$;

7. $M, w=\square \phi \Leftrightarrow\left(w, w^{\prime}\right) \in R$ implies $M, w^{\prime} \models \phi$ for any $w^{\prime}$ in $W$.

The following lemma holds immediately:

Lemma 2.1. For any Kripke model $M=(W, R, D, I)$, the underlying frame $(W, R)$ is of bounded length if and only if for any $w \in W$ there exist some $n \in \mathbb{N}$ such that $M, w \models \neg \diamond^{n} \top$.

Validity of a non-closed formula is defined by the validity of the universal closure of it. Let $\phi$ be a formula. If every world $w$ in a Kripke model $N$ satisfies $M, w \models \phi$, we write $M \models \phi$. If every Kripke model $M$ over a frame $F$ satisfies $M=\phi$, we write $F \mid \phi$. If every $F$ in a class $C$ of Kripke frames satisfies $F \models \phi$, we write $C \models \phi$.

\section{Non-compact proof system for predicate extension of the logic of provability}

In this section, we introduce a proof system NQGL for a predicate extension of GL. The proof system NQGL is a variant of Gentzen-style sequent calculus. A sequent $\Gamma \rightarrow \Delta$ is defined to be a pair of finite sets $\Gamma$ and $\Delta$ of formulas. The axiom schemta of NQGL are $p \rightarrow p, \rightarrow \top, \perp \rightarrow$, and the derivation rules of NQGL are the following:

Set

$$
\frac{\Gamma \rightarrow \Delta}{\Gamma^{\prime} \rightarrow \Delta^{\prime}} \quad\left(\text { where } \Gamma \subseteq \Gamma^{\prime} \text { and } \Delta \subseteq \Delta^{\prime}\right)
$$

Cut

$$
\frac{\Gamma \rightarrow \Delta, \phi \quad \phi, \Lambda \rightarrow \Xi}{\Gamma, \Lambda \rightarrow \Delta, \Xi}
$$

Conjunction

$$
\frac{\Gamma \rightarrow \Delta, \phi \quad \Gamma \rightarrow \Delta, \psi}{\Gamma \rightarrow \Delta, \phi \wedge \psi} \quad \frac{\phi, \Gamma \rightarrow \Delta}{\phi \wedge \psi, \Gamma \rightarrow \Delta} \quad \frac{\psi, \Gamma \rightarrow \Delta}{\phi \wedge \psi, \Gamma \rightarrow \Delta}
$$


Implication

$$
\frac{\phi, \Gamma \rightarrow \Delta, \psi}{\Gamma \rightarrow \Delta, \phi \supset \psi} \quad \frac{\Gamma \rightarrow \Delta, \phi \quad \psi, \Lambda \rightarrow \Xi}{\phi \supset \psi, \Gamma, \Lambda \rightarrow \Delta, \Xi}
$$

Negation

$$
\frac{\phi, \Gamma \rightarrow \Delta}{\Gamma \rightarrow \Delta, \neg \phi} \quad \frac{\Gamma \rightarrow \Delta, \phi}{\neg \phi, \Gamma \rightarrow \Delta}
$$

For all

$$
\frac{\Gamma \rightarrow \Delta, \phi[y / x]}{\Gamma \rightarrow \Delta, \forall x \phi} \quad \frac{\phi[z / x], \Gamma \rightarrow \Delta}{\forall x \phi, \Gamma \rightarrow \Delta}
$$

Here, $y$ is a variable in $\mathcal{V}$ which does not occur in any formulas in the lower sequent, and $z$ is any variable in $\mathcal{V}$.

Box

$$
\frac{\square \Gamma, \Delta \rightarrow \phi}{\square \Gamma, \square \Delta \rightarrow \square \phi}
$$

\section{Boundedness of length}

$$
\frac{\Gamma \rightarrow \Delta, \diamond^{n} \uparrow \quad(\text { for any } n \in \mathbb{N})}{\Gamma \rightarrow \Delta}
$$

Here, the set of upper sequents is countably infinite.

For any sequent $\Gamma \rightarrow \Delta$, we write $\vdash_{\text {NQGL }} \Gamma \rightarrow \Delta$ if it is derivable in NQGL. A formula $\phi$ is said to be derivable in NQGL, if $\vdash_{\mathrm{NQGL}} \rightarrow \phi$. If this is the case, we write $\vdash_{\text {NQGL }} \phi$. It is easy to see that the rule Box is equivalent to $\square p \supset \square \square p$ plus standard necessitation rule

$$
\frac{\Gamma \rightarrow \phi}{\square \Gamma \rightarrow \square \phi} .
$$

The rule Boundedness of length denotes that

$$
\bigwedge_{n \in \mathbb{N}} \diamond^{n} 1=0
$$

holds in the Lindenbaum algebra of the logic defined by NQGL. Note that if a Boolean algebra with operators satisfies (4), the following equation holds in it, either:

$$
\bigwedge_{n \in \mathbb{N}} \square \diamond^{n} 1=\square 0 .
$$

Theorem 3.1. (Soundness of NQGL). If $\vdash_{\mathrm{NQGL}} \phi$, then $\mathfrak{B} \mathfrak{L} \models \phi$, for any formula $\phi$. 


\section{Finitely consistent pairs and saturated pairs}

In this section, we introduce some notions which are used to show the Kripke completeness and the admissibility of the cut-rule for NQGL. We write $\mathrm{NQGL}^{-}$for the cut-free fragment of NQGL, and $\vdash_{\mathrm{NQGL}^{-}} \Gamma \rightarrow \Delta$ if a sequent $\Gamma \rightarrow \Delta$ is derivable in $\mathrm{NQGL}^{-}$.

Definition 4.1. A pair $(S, T)$ of sets of formulas is said to be finitely consistent if for any finite sets $S^{\prime} \subseteq S$ and $T^{\prime} \subseteq T$,

$$
\nvdash_{\mathrm{NQGL}^{-}} S^{\prime} \rightarrow T^{\prime}
$$

Definition 4.2. Let $\mathcal{U}$ be a set of variables. A finitely consistent pair $(S, T)$ of subsets of $\Phi(\mathcal{U})$ is said to be $\mathcal{U}$-saturated, if the following conditions are satisfied:

1. If $\phi_{1} \wedge \phi_{2} \in S$, then $\phi_{1}, \phi_{2} \in S$, and if $\phi_{1} \wedge \phi_{2} \in T$, then either $\phi_{1} \in T$ or $\phi_{2} \in T$.

2. If $\phi_{1} \supset \phi_{2} \in S$, then either $\phi_{1} \in T$ or $\phi_{2} \in S$, and if $\phi_{1} \supset \phi_{2} \in T$, then $\phi_{1} \in S$ and $\phi_{2} \in T$.

3. If $\neg \phi \in S$, then $\phi \in T$, and if $\neg \phi \in T$, then $\phi \in S$.

4. If $\forall x \phi \in S$, then $\phi[z / x] \in S$ for all $z \in \mathcal{U}$, and if $\forall x \phi \in T$, then $\phi[z / x] \in T$ for some $z \in \mathcal{U}$.

Definition 4.3. A finitely consistent pair $(S, T)$ of formulas is called a GL-pair, if $\square \neg \diamond^{n} \top \in S$ for some $n \in \mathbb{N}$.

Theorem 4.4. Let $\mathcal{U}$ be a coinfinite subset of $\mathcal{V}$. Suppose $(S, T)$ is a finitely consistent pair of subsets of $\Phi(\mathcal{U})$. Then, there exists a coinfinite subset $\mathcal{U}^{\prime}$ of $\mathcal{V}$ and a $\mathcal{U}^{\prime}$-saturated pair $\left(S^{\prime}, T^{\prime}\right)$ such that $\mathcal{U} \subseteq \mathcal{U}^{\prime}, S \subseteq S^{\prime}$, and $T \subseteq T^{\prime}$.

Proof. Take a coinfinite subset $\mathcal{W}$ of $\mathcal{V}$ such that $\mathcal{U}$ is a coinfinite subset of $\mathcal{W}$. Let $\left(\phi_{n}\right)_{n \in \mathbb{N}}$ be a sequence of formulas of $\Phi(\mathcal{W})$ such that each formula of $\Phi(\mathcal{W})$ occurs infinitely many times in it. For example, if $\left(\gamma_{n}\right)_{n \in \mathbb{N}}$ is an enumeration of all formulas of $\Phi(\mathcal{W}),\left(\phi_{n}\right)_{n \in \mathbb{N}}$ could be

$$
\gamma_{0}, \gamma_{0}, \gamma_{1}, \gamma_{0}, \gamma_{1}, \gamma_{2}, \gamma_{0}, \gamma_{1}, \gamma_{2}, \gamma_{3}, \cdots
$$

Define lists $\left(\mathcal{U}_{n}\right)_{n \in \mathbb{N}}$ and $\left(\left(S_{n}, T_{n}\right)\right)_{n \in \mathbb{N}}$ which satisfies the following: 
1. for every $n \in \mathbb{N}, \mathcal{U}_{n}$ is a coinfinite subset of $\mathcal{W}$ and $\mathcal{U}_{n} \subseteq \mathcal{U}_{n+1}$;

2. for every $n \in \mathbb{N},\left(S_{n}, T_{n}\right)$ is a finitely consistent pair of subsets of $\Phi\left(\mathcal{U}_{n}\right), S_{n} \subseteq S_{n+1}$, and $T_{n} \subseteq T_{n+1}$.

First, let $\mathcal{U}_{0}=\mathcal{U}$ and $\left(S_{0}, T_{0}\right)=(S, T)$. Suppose $\mathcal{U}_{i}$ and $\left(S_{i}, T_{i}\right)$ are defined for every $i \leqq n$ :

- Case $\phi_{n}=\psi_{1} \wedge \psi_{2}$ : Define $\mathcal{U}_{n+1}$ by $\mathcal{U}_{n}$. If $\psi_{1} \wedge \psi_{2} \in S_{n}$, then define $\left(S_{n+1}, T_{n+1}\right)$ by $\left(S_{n} \cup\left\{\psi_{1}, \psi_{2}\right\}, T_{n}\right)$. If $\psi_{1} \wedge \psi_{2} \in T_{n}$, then define $\left(S_{n+1}, T_{n+1}\right)$ by $\left(S_{n}, T_{n} \cup\left\{\psi_{1}\right\}\right)$ or by $\left(S_{n}, T_{n} \cup\left\{\psi_{2}\right\}\right)$, so that $\left(S_{n+1}, T_{n+1}\right)$ is finitely consistent.

- Case $\phi_{n}=\psi_{1} \supset \psi_{2}$ : Define $\mathcal{U}_{n+1}$ by $\mathcal{U}_{n}$. If $\psi_{1} \supset \psi_{2} \in S_{n}$, then define $\left(S_{n+1}, T_{n+1}\right)$ by $\left(S_{n}, T_{n} \cup\left\{\psi_{1}\right\}\right)$ or by $\left(S_{n} \cup\left\{\psi_{2}\right\}, T_{n}\right)$, so that $\left(S_{n+1}, T_{n+1}\right)$ is finitely consistent. If $\psi_{1} \supset \psi_{2} \in T_{n}$, then define $\left(S_{n+1}, T_{n+1}\right)$ by $\left(S_{n} \cup\left\{\psi_{1}\right\}, T_{n} \cup\left\{\psi_{2}\right\}\right)$.

- Case $\phi_{n}=\neg \psi$ : Define $\mathcal{U}_{n+1}$ by $\mathcal{U}_{n}$. If $\neg \psi \in S_{n}$, then define $\left(S_{n+1}, T_{n+1}\right.$ ', by $\left(S_{n}, T_{n} \cup\{\psi\}\right)$. If $\neg \psi \in T_{n}$, then define $\left(S_{n+1}, T_{n+1}\right)$ by $\left(S_{n} \cup\right.$ $\left.\{\psi\}, T_{n}\right)$.

- Case $\phi_{n}=\forall x \psi$ : If $\forall x \psi \in S_{n}$, then define $\mathcal{U}_{n+1}$ by $\mathcal{U}_{n}$, and define $\left(S_{n+1}, T_{n+1}\right)$ by $\left(S_{n} \cup\left\{\psi[z / x] \mid z \in \mathcal{U}_{n}\right\}, T_{n}\right)$. If $\forall x \psi \in T_{n}$, then define $\mathcal{U}_{n+1}$ by $\mathcal{U}_{n} \cup\{z\}$, where $z \in \mathcal{W} \backslash \mathcal{U}_{n}$, and define $\left(S_{n+1}, T_{n+1}\right)$ by $\left(S_{n}, T_{n} \cup\{\psi[z / x]\}\right)$.

- Otherwise, $\mathcal{U}_{n+1}=\mathcal{U}_{n}$ and $\left(S_{n}, T_{n}\right)=\left(S_{n+1}, T_{n+1}\right)$.

It is clear that the conditions 1 and 2 are satisfied. Now, Let

$$
\mathcal{U}^{\prime}=\bigcup_{n \in \mathbb{N}} \mathcal{U}_{n}, S^{\prime}=\bigcup_{n \in \mathbb{N}} S_{n}, T^{\prime}=\bigcup_{n \in \mathbb{N}} T_{n} .
$$

Since each formula in $\Phi(\mathcal{W})$ occurs infinitely many times in the list $\left(\phi_{n}\right)_{n \in \mathbb{N}}$, $\mathcal{U}^{\prime}$ and $\left(S^{\prime}, T^{\prime}\right)$ satisfy the first part of the 4 th condition of Definition 4.2. It is easy to check the other conditions are fulfilled.

Theorem 4.5. Let $\mathcal{U}$ be a coinfinite subset of $\mathcal{V}$ and $(S, T)$ a $\mathcal{U}$-saturated GL-pair. If $\square \phi \in T$, there exists a coinfinite subset $\mathcal{U}^{\prime}$ of $\mathcal{V}$ and a $\mathcal{U}^{\prime}$-saturated GL-pair $\left(S^{\prime}, T^{\prime}\right)$ such that $\mathcal{U} \subseteq \mathcal{U}^{\prime}, \phi \in T^{\prime}$, and $\square^{-1} S \cup \square \square^{-1} S \subseteq S^{\prime}$. 
Proof. Since $(S, T)$ is finitely consistent, so is $\left(\square^{-1} S \cup \square \square^{-1} S,\{\phi\}\right)$. Since $(S, T)$ is a GL-pair, $\square \neg \nabla^{n} T \in \square^{-1} S$ for some $n \in \mathbb{N}$. Now, by Theorem 4.4, there exists a coinfinite subset $\mathcal{U}^{\prime}$ of $\mathcal{V}$ and $\mathcal{U}^{\prime}$-saturated pair $\left(S^{\prime}, T^{\prime}\right)$ such that $\mathcal{U} \subseteq \mathcal{U}^{\prime}, \phi \in T^{\prime}$, and $\square^{-1} S \cup \square \square^{-1} S \subseteq S^{\prime}$.

\section{Kripke completeness of $\mathrm{NQGL}^{-}$}

In this section, we show that the cut-free fragment $\mathrm{NQGL}^{-}$of $\mathrm{NQGL}$ is Kripke complete with respect to $\mathfrak{B L}$. The admissibility of the cut-rule follows from the completeness theorem and Theorem 3.1.

Theorem 5.1. If $\forall_{\mathrm{NQGL}^{-}} \Gamma \rightarrow \Delta$, there exists a coinfinite subset $\mathcal{U}$ of $\mathcal{V}$ and a $\mathcal{U}$-saturated $\mathbf{G L}$-pair $(S, T)$ such that $\Gamma \subseteq S$ and $\Delta \subseteq T$.

Proof. By the rule of boundedness, there exists $n \in \mathbb{N}$ such that

$$
\forall_{\mathrm{NQGL}^{-}} \square \neg \diamond^{n} \mathrm{\top}, \Gamma \rightarrow \Delta .
$$

Apply Theorem 4.4 to $\operatorname{Var}(\Gamma \cup \Delta)$ and $\left(\left\{\square \neg \diamond^{n} \top\right\} \cup \Gamma, \Delta\right)$.

Theorem 5.2. (Kripke completeness of $\mathrm{NQGL}^{-}$). A formula $\phi$ is derivable in $\mathrm{NQGL}^{-}$if and only if $\mathfrak{B} \mathfrak{L}=\phi$.

Proof. We only show the if-part. Define a model $M=(W, R, D, I)$ as follows:

- $W$ is the set of all triples $(\mathcal{U}, S, T)$, where $\mathcal{U}$ is a coinfinite subset of $\mathcal{V}$ and $(S, T)$ is a $\mathcal{U}$-saturated GL-pair.

- For any $(\mathcal{U}, S, T)$ and $\left(\mathcal{U}^{\prime}, S^{\prime}, T^{\prime}\right)$ in $W$, $\left((\mathcal{U}, S, T),\left(\mathcal{U}^{\prime}, S^{\prime}, T^{\prime}\right)\right) \in R \Leftrightarrow \mathcal{U} \subseteq \mathcal{U}^{\prime}$ and $\square^{-1} S \cup \square \square^{-1} S \subseteq S^{\prime}$.

- For any $(\mathcal{U}, S, T) \in W, D_{(\mathcal{U}, S, T)}=\mathcal{U}$.

- For any $(\mathcal{U}, S, T) \in W$ and any predicate symbol $P$ of arity $n$,

$$
I((\mathcal{U}, S, T), P)=\left\{\left(x_{1}, \ldots, x_{n}\right) \in \mathcal{V}^{n} \mid P\left(x_{1}, \ldots, x_{n}\right) \in S\right\} .
$$


By definition of $R$, the frame $(W, R)$ is transitive. We claim that for any formula $\phi$ and $(\mathcal{U}, S, T) \in W$,

$$
\phi \in S \Rightarrow M,(\mathcal{U}, S, T) \models \phi, \quad \phi \in T \Rightarrow M,(\mathcal{U}, S, T) \forall \varnothing .
$$

We prove the claim only for the cases of $\phi=P\left(x_{1}, \ldots, x_{n}\right), \forall x \psi(x)$, and $\square \psi$ :

- Case $\phi=P\left(x_{1}, \ldots, x_{n}\right)$ : By definitions of $I$ and $\models$,

$$
\begin{aligned}
P\left(x_{1}, \ldots, x_{n}\right) \in S & \Leftrightarrow \quad\left(x_{1}, \ldots, x_{n}\right) \in I((\mathcal{U}, S, T), P) \\
& \Leftrightarrow \quad M,(\mathcal{U}, S, T) \models P\left(x_{1}, \ldots, x_{n}\right) .
\end{aligned}
$$

Since $(S, T)$ is finitely consistent,

$$
\begin{aligned}
P\left(x_{1}, \ldots, x_{n}\right) \in T & \Rightarrow P\left(x_{1}, \ldots, x_{n}\right) \notin S \\
& \Leftrightarrow\left(x_{1}, \ldots, x_{n}\right) \notin I((\mathcal{U}, S, T), P) \\
& \Leftrightarrow M,(\mathcal{U}, S, T) \notin P\left(x_{1}, \ldots, x_{n}\right) .
\end{aligned}
$$

- Case $\phi=\forall x \psi(x)$ : If $\forall x \psi(x) \in S$, then $\psi(z) \in S$ for any $z \in \mathcal{U}$, since $(S, T)$ is $\mathcal{U}$-saturated. Hence, by induction hypothesis, $M,(\mathcal{U}, S, T) \models$ $\psi(z)$ for any $z \in D_{(\mathcal{U}, S, T)}$. If $\forall x \psi(x) \in T$, then, $\psi(z) \in T$ for some $z \in \mathcal{U}$, since $(S, T)$ is $\mathcal{U}$-saturated. By induction hypothesis, $M,(\mathcal{U}, S, T) \not \forall \psi(z)$ for some $z \in D_{(\mathcal{U}, S, T)}$.

- Case $\phi=\square \psi$ : Suppose $\square \psi \in S$ and $\left((\mathcal{U}, S, T),\left(\mathcal{U}^{\prime}, S^{\prime}, T^{\prime}\right)\right) \in R$. Then $\psi \in S^{\prime}$ by definition of $R$. By induction hypothesis, $M,\left(\mathcal{U}^{\prime}, S^{\prime}, T^{\prime}\right) \models$ $\psi$. Suppose $\square \psi \in T$. Then, by Theorem 4.5, there exists a coinfinite subset $\mathcal{U}^{\prime}$ of $\mathcal{V}$ and a $\mathcal{U}^{\prime}$-saturated GL-pair $\left(S^{\prime}, T^{\prime}\right)$ such that $\mathcal{U} \subseteq \mathcal{U}^{\prime}, \psi \in T^{\prime}$, and $\square^{-1} S \cup \square \square^{-1} S \subseteq S^{\prime}$. Then, $\left(\mathcal{U}^{\prime}, S^{\prime}, T^{\prime}\right) \in$ $W,\left((\mathcal{U}, S, T),\left(\mathcal{U}^{\prime}, S^{\prime}, T^{\prime}\right)\right) \in R$, and, by the induction hypothesis, $M,\left(\mathcal{U}^{\prime}, S^{\prime}, T^{\prime}\right) \forall \neq \psi$.

This completes the proof of the claim. By using the claim and Lemma 2.1, $(W, R) \in \mathfrak{B} \mathfrak{L}$. Now, suppose $\forall_{\mathrm{NQGL}^{-}} \Gamma \rightarrow \Delta$. Then, by Theorem 5.1, there exists $(\mathcal{U}, S, T) \in W$ such that $\Gamma \subseteq S$ and $\Delta \leqq T$. Hence, $M,(\mathcal{U}, S, T) \not \models \Gamma \rightarrow \Delta$ by the claim.

Remark. It is not clear whether non-compactness is necessary to axiomatize $\mathbf{M Q}(\mathfrak{B L})$ or not. Researches from a similar viewpoint can be 
found in common knowledge logic. While the logic CKL, the propositional common knowledge logic defined by the class of Kripke frames, is recursively axiomatizable ([5]), the logic QCKL, the predicate common knowledge logic defined by the class of Kripke frames with constant domains, is not ([14]). Therefore, simple predicate extension of any recursive proof systems for CKL does not axiomatize QCKL. On the other hand, by adopting non-compactness, it is possible to give proof systems for QCKL $([11,12])$, and one of them in $[12]$ is cut-free. It might be an interesting problem whether the same phenomenon can be found in predicate provability logic or not.

\section{Acknowledgements}

The author would like to thank Taishi Kurahashi, Katsumi Sasaki, and Tatsuya Shimura for their helpful comments and suggestions.

\section{References}

[1] S. Artemov and G. Dzhaparidze, Finite Kripke models and predicate logics of provability, The Journal of Symbolic Logic 55 (1990), 1090-1098.

[2] A. Avron, On modal systems having arithmetical interpretations, The Journal of Symbolic Logic 49 (1984), 935-942.

[3] G. Boolos, The logic of provability, Cambridge University Press, 1993.

[4] R. Goldblatt, Mathematics of Modality, vol. 43 of CSLI Lecture Notes, CSLI Publications, Stanford, 1993.

[5] J.Y. Halpern and Y. Moses, A guide to completeness and complexity for modal logics of knowledge and beliefs, Artificial Intelligence 54 (1992), 319-379.

[6] T. Kurahashi, Arithmetical interpretations and Kripke frames of predicate modal logic of provability, The Review of Symbolic Logic 6 (2013), 129-146.

[7] D. Leivant, On the proof theory of modal logic for arithmetic provability, The Journal of Symbolic Logic 46 (1981), 531-538.

[8] F. Montagna, The predicate modal logic of provability, Notre Dame Journal of Formal Logic 25 (1984), 179-189.

[9] Y. Schwarz and G. Tourlakis, On the proof-theory of a first-order extension of GL, Logic and Logical Philosophy 23 (2014), 329-363.

[10] K. Segerberg, A model existence theorem in infinitary propositional modal logic, Journal of Philosophical Logic 23 (1994), 337-367. 
[11] Y. Tanaka, Model existence in non-compact modal logic, Studia Logica 67 (2001), $61-73$.

[12] Y. Tanaka, Some proof systems for predicate common knowledge logic, Reports on Mathematical Logic 37 (2003), 79-100.

[13] S. Valentini, The modal logic of provability, Journal of Philosophical Logic 12 (1983), 471-476.

[14] F. Wolter, First order common knowledge logics, Studia Logica 65 (2000), 249-271.

[15] R.E. Yavorsky, On arithmetical completeness of first order logics of provability, In: F. Wolter, H. Wansin and M. Zakharyaschev, editors, vol. 3 of Advances in Modal Logic, CSLI Publications, 2001, pp. 1-16.

Kyushu Sangyo University

2-3-1 Matsukadai, Higashi-ku

Fukuoka 813-8503, Japan

ytanaka@ip.kyusan-u.ac.jp 